\title{
Reduction of colonic inflammation in HLA-B27 transgenic rats by feeding Marie Ménard apples, rich in polyphenols $\dagger$
}

\author{
Cinzia Castagnini ${ }^{1}$, Cristina Luceri ${ }^{1}$, Simona Toti ${ }^{1,2}$, Elisabetta Bigagli ${ }^{1}$, Giovanna Caderni $^{1}$, \\ Angelo P. Femia ${ }^{1}$, Lisa Giovannelli ${ }^{1}$, Maura Lodovici ${ }^{1}$, Vanessa Pitozzi ${ }^{1}$, Maddalena Salvadori ${ }^{1}$, \\ Luca Messerini $^{3}$, Rocio Martin ${ }^{4}$, Erwin G. Zoetendal ${ }^{4}$, Stan Gaj ${ }^{5,6}$, Lars Eijssen ${ }^{5}$, Chris T. Evelo ${ }^{5}$, \\ Catherine M. G. C. Renard ${ }^{7}$, Alain Baron ${ }^{7}$ and Piero Dolara ${ }^{1}$ \\ ${ }^{1}$ Department of Preclinical and Clinical Pharmacology, University of Florence, Florence, Italy \\ ${ }^{2}$ Department of Statistics, University of Florence, Florence, Italy \\ ${ }^{3}$ Department of Pathology, University of Florence, Florence, Italy \\ ${ }^{4}$ Laboratory of Microbiology, Wageningen University, Wageningen, The Netherlands \\ ${ }^{5}$ Department of Bioinformatics - BiGCaT, Maastricht University, Maastricht, The Netherlands \\ ${ }^{6}$ Nutrigenomics Consortium, Top Institute Food and Nutrition, Wageningen, The Netherlands \\ ${ }^{7}$ UR117 Cidricoles - Biotransformation des Fruits et Légumes, INRA, Le Rheu, France
}

(Received 28 January 2009 - Revised 13 May 2009 - Accepted 3 June 2009 - First published online 22 July 2009)

Inflammatory bowel diseases (IBD) are immunomediated ailments affecting millions of individuals. Although diet is regarded as an important factor influencing IBD, there are no accepted dietary recommendations presently available. We administered 7.6\% lyophilised apples obtained from two cultivars (Golden Delicious and Marie Ménard, low and high in polyphenols, respectively) to HLA-B27 transgenic rats which develop spontaneous IBD. After 3 months feeding, rats fed Marie Ménard apples had reduced myeloperoxidase activity (3.6 (SEM 0·3) v. $2 \cdot 2$ (SEM 0·2) U/g tissue; $P<0.05)$ and reduced cyclo-oxygenase-2 $(P<0.05)$ and inducible NO synthase gene expression $(P<0 \cdot 01)$ in the colon mucosa and significantly less diarrhoea $(P<0.05)$, compared with control rats. Cell proliferation in the colon mucosa was reduced significantly by feeding Golden Delicious apples, with a borderline effect of Marie Ménard apples. Gene expression profiling of the colon mucosa, analysed using the Whole Rat Genome $4 \times 44 \mathrm{~K}$ Agilent Arrays, revealed a down-regulation of the pathways of PG synthesis, mitogen-activated protein kinase (MAPK) signalling and TNF $\alpha-N F-\kappa B$ in Marie Ménard-fed rats. In the stools of the animals of this group we also measured a significant reduction of bacteria of the Bacteriodes fragilis group. In conclusion, the administration of Marie Ménard apples, rich in polyphenols and used at present only in the manufacturing of cider, ameliorates colon inflammation in transgenic rats developing spontaneous intestinal inflammation, suggesting the possible use of these and other apple varieties to control inflammation in IBD patients.

Apples: Microarray analysis: Expression profiles: Bacteriodes fragilis

Inflammatory bowel diseases (IBD), such as Crohn's disease and ulcerative colitis, are increasing in prevalence in southern Europe, Asia and much of the developing world, while their occurrence is stable in high-incidence areas such as northern Europe and North America. It has been estimated that 1.4 million individuals in the USA and 2.2 million individuals in Europe alone suffer from these diseases ${ }^{(1)}$.

The aetiology of IBD is still unknown, although genetic background and modern lifestyle seem to be important factors $^{(2)}$. Diet also plays a role in the development or clinical course of IBD, with meat and alcoholic beverages increasing the likelihood of relapse in ulcerative colitis patients ${ }^{(3)}$. On the other hand, elemental diet (liquid nutrition where $\mathrm{N}$ is derived from free amino acids, sugars from simple digestible carbohydrates and fat from TAG) has been shown to have beneficial effects ${ }^{(4)}$. However, elemental diets are unpalatable and can only be used for limited periods of time due to patient non-compliance and side-effects ${ }^{(5)}$. In practice, there are no effective and acceptable long-term dietary recommendations for IBD.

Phytochemicals, such as polyphenols from fruits and vegetables (i.e. proanthocyanidins), modulate cellular signalling processes, exert anti-inflammatory and antioxidant activity and modify intestinal microflora ${ }^{(6-8)}$. Apples contain an exceptional selection of such bioactive phytochemicals. However, most of the studies on apples have focused on single compounds, underestimating the effects related to the combination of a complex phytochemical mixture. On this basis, we wanted to verify whether consumption of foods such as apples might assist in controlling intestinal inflammation in rats.

\footnotetext{
Abbreviations: COX2, cyclo-oxygenase-2; DGGE, denaturing gradient gel electrophoresis; IBD, inflammatory bowel disease.

* Corresponding author: Dr Cristina Luceri, fax + 39 0554271280, email cristina.luceri@unifi.it

$\dagger$ Microarray data are available from ArrayExpress (http://www.ebi.ac.uk/microarray-as/ae/), accession E-TABM-514.
} 
A variety of experimental models are available for studies on chronic intestinal inflammation in vivo. One of these is the intermittent administration of dextrane sulfate sodium, which damages epithelial cell junctions and makes the mucosa permeable to intestinal bacteria $^{(9)}$; however, the dextrane sulfate sodium model is not clearly correlated with spontaneous human IBD.

HLA-B27 transgenic rats, used in the present study, derive from F344 rats and express human $\beta 2$-microglobulin and the histocompatibility gene HLA-B27; they spontaneously develop immune-mediated intestinal inflammation, resembling histologically human $\mathrm{IBD}^{(10)}$. The microbial intestinal environment influences colitis in HLA-B27 rats and intestinal inflammation is not observed in germ-free environments ${ }^{(11)}$. In this rat model colitis is induced by administering Bacteroides vulgatus, while Escherichia coli is inactive ${ }^{(12)}$. Additionally, in HLA-B27 rats, the colitis is mediated by $\mathrm{CD}^{+}$but not by $\mathrm{CD}^{+}$cells, suggesting a complex host-microbiota interaction in which genetic host factors play a crucial role ${ }^{(13)}$.

The aim of the present study was to verify whether the addition to the diet of two apple varieties, Marie Ménard and Golden Delicious, with different polyphenol composition, could reduce intestinal inflammation in HLA-B27 rats and consequently ameliorate colitis. This topic was addressed through the characterisation of classical inflammation markers, faecal microbiota content and colon mucosa whole-genome expression profiles.

\section{Materials and methods}

\section{Apple powder production and characterisation}

Cider apple fruits (Malus domestica Borkh.) from the Marie Ménard variety were harvested at maturity during the 2002 season in the experimental orchard of the Centre Technique des Productions Cidricoles (Sées, Orne, France). Golden Delicious apples were bought from a local wholesale grocery in the same location.

The apple powder used in the feeding experiments contained complete apples (skin and pulp) and was prepared from apples frozen in liquid $\mathrm{N}_{2}$ after slicing, and freezedried. After freeze-drying, samples were blended in a food processor and portioned in special hermetically sealed bags to avoid storage degradation. All samples were stored at $20^{\circ} \mathrm{C}$ until analysis or diet preparation.

Polyphenols were measured by HPLC after thioacidolysis as previously described ${ }^{(14)}$. The average degree of polymerisation was measured by calculating the molar ratio of all the flavan-3-ol units (thioether adducts plus terminal units) to $(-)$-epicatechin and $(+)$-catechin corresponding to terminal units. The HPLC apparatus was a Waters (Milford, MA, USA) system 717 plus autosampler equipped with a cooling module set at $4{ }^{\circ} \mathrm{C}$, a $600 \mathrm{E}$ multisolvent system, a 996 photodiode array detector, and a Millennium 2010 Manager system. The column was a Purospher RP18 endcapped, $5 \mu \mathrm{m}$ (Merck, Darmstadt, Germany). The solvent system was a gradient of solvent A (aqueous acetic acid, $2.5 \% \mathrm{v} / \mathrm{v}$ ) and solvent B (acetonitrile): initial composition $3 \% \mathrm{~B}$; linear gradient to $9 \% \mathrm{~B}$ from 0 to $5 \mathrm{~min}$; linear gradient to $16 \% \mathrm{~B}$ from 5 to $15 \mathrm{~min}$; linear gradient to $50 \% \mathrm{~B}$ from 15 to $45 \mathrm{~min}$ followed by washing and reconditioning the column.
$\mathrm{N}$ was analysed by the Kjeldahl method. Proteins were calculated as $\mathrm{N} \times 6 \cdot 25^{(15)}$.

Cell walls were quantified as alcohol-insoluble solids from the freeze-dried apple powders, as described elsewhere ${ }^{(16)}$. Starch was quantified by an enzymic-colorimetric method ${ }^{(17)}$ as described previously ${ }^{(18)}$. Sugars (glucose, fructose and sucrose) and L-malic acid were determined by enzymic assays by Boehringer Mannheim/R-Biopharm (R-Biopharm AG, Darmstadt, Germany) according to the producer's specifications.

\section{Animals and diets}

The experimental protocol, designed in conformity with the recommendations of the European Economic Community (86/609/CEE) for the care and the use of laboratory animals, in agreement with the Good Laboratory Practices, was approved by the animal care committee of the University of Florence (Italy).

Male HLA-B27 transgenic rats (Taconic Laboratory, Germantown, NY, USA), aged 6-8 weeks, were maintained in specific pathogen-free condition (but not germ-free conditions) and divided into three groups: controls ( $n$ 6); rats fed lyophilised Golden Delicious apples ( $n$ 7); rats fed lyophilised Marie Ménard apples (n 7). Dietary components (Piccioni, Gessate, Milan, Italy) were based on the American Institute of Nutrition (AIN)-76 diet, modified to contain high fat $\left(23 \%\right.$ maize oil, w/w) and low cellulose $(2 \%, \mathrm{w} / \mathrm{w})^{(19)}$. The apple-supplemented diets contained $7.6 \%$ of lyophilised apple pulp and were modified to compensate for sucrose and fibres in the apple pulp, deriving from Golden Delicious apples or Marie Ménard apples (Tables 1 and 2).

After 12 weeks of treatment the animals were killed. For each animal, the last part of the distal colon was fixed in buffered formalin for histological analysis. The remaining part of the distal colon was scraped with a glass slide to isolate the mucosal layer and stored at $-80^{\circ} \mathrm{C}$ for DNA extraction and biochemical determinations and in RNAlater (Qiagen, Milan, Italy) for RNA analyses.

\section{Histological grading of colitis}

The extent of tissue damage was determined by assigning a numerical score to colon tissue sections obtained from

Table 1. Composition of lyophilised Marie Ménard and Golden Delicious apples $(\mathrm{mg} / \mathrm{g})$

\begin{tabular}{lcc}
\hline & Golden Delicious & Marie Ménard \\
\hline Fibres & 203.5 & 193.3 \\
Hydroxycinnamic acids & & \\
$\quad$ Caffeoylquinic acid & 0.49 & 6.06 \\
$\quad$-Coumarylquinic acid & 0.04 & 0.25 \\
Flavan-3-ols & & \\
$\quad$ Monomers & 0.43 & 2.78 \\
$\quad$ Proanthocyanidins & 4.03 & 26.07 \\
Degree of polymerisation & 1.98 & 3.60 \\
Dihydrochalcones & 0.38 & 0.62 \\
Flavonols & 0.05 & 0.30 \\
Starch & 0.6 & 30.1 \\
Glucose & 90 & 83 \\
Fructose & 404 & 376 \\
Sucrose & 198 & 187 \\
Malic acid & 20 & 11 \\
\hline
\end{tabular}


Table 2. Composition of experimental diets $(\mathrm{g} / \mathrm{kg})$

\begin{tabular}{lcc}
\hline & Control diet & Apple diets \\
\hline Lyophilised apples & - & 76 \\
Maize oil & 230 & 230 \\
Sucrose & 322 & 271 \\
Starch & 101 & 101 \\
Casein & 230 & 230 \\
Cellulose & 60 & 44.9 \\
Mineral mix AIN-76 & 40 & 40 \\
Vitamin mix AIN-76 & 12 & 12 \\
Choline & 2 & 2 \\
DL-Methionine & 3 & 3 \\
\hline
\end{tabular}

AIN, American Institute of Nutrition.

each rat. Full-thickness sections of fixed colons were stained with haematoxylin-eosin and scored blindly by a pathologist according to established criteria for characterisation of inflammation $(0=$ normal, $1=$ local neutrophil infiltration, $2=$ loss of glands due to inflammation, $3=$ moderate ulceration or foci with the loss of more than five glands, $4=$ extensive ulcerations). Mucosal injury was also assessed $(0=$ normal, $1=$ distortion and/or destruction of the lower third of the glands, 2 = distortion and/or destruction of the lower twothirds of the glands with remaining surface epithelium, $3=$ loss of entire glands). A 'colitis score' of each rat was calculated as the sum of the two sub-scores. Results were expressed as average colitis score in each experimental group.

\section{Faecal water content}

Fresh faecal samples were harvested when the rats were killed and frozen at $-80^{\circ} \mathrm{C}$ until analyses. To determine the degree of diarrhoea, faecal samples were dried in a dehumidified oven at $50^{\circ} \mathrm{C}$ until stable in weight and the water content was expressed as percentage of the fresh faecal weight.

\section{Faecal microbiota analysis}

To assess the effect of the treatments on intestinal microbiota composition, PCR-denaturing gradient gel electrophoresis (DGGE) and real-time PCR were performed on faecal DNA extracted according to a previously described method ${ }^{(20)}$. PCR amplification of total bacteria, Lactobacillus, Bifidobacterium and Bacteroides was carried out by targeting $16 \mathrm{~S}$ rRNA gene sequences, using primers and PCR conditions as described previously ${ }^{(21-24)}$. DGGE analysis of PCR products was performed using the DCode System (Bio-Rad Laboratories, Hercules, CA, USA). Polyacrylamide denaturating gels were poured from the top using a gradient maker and a pump (Econopump; Bio-Rad, La Jolla, CA, USA); gradients of $30-60 \%, 35-55 \%, 40-50 \%$ and $30-50 \%$ were used for separation of specific amplicons for total bacteria, Lactobacillus, Bifidobacterium and Bacteroides, respectively. Electrophoresis was performed for $16 \mathrm{~h}$ at $85 \mathrm{~V}$ in a $0.5 \times$ TAE (Tris-acetate-EDTA) buffer at $60^{\circ} \mathrm{C}$; gels were stained with $\mathrm{AgNO}_{3}^{(25)}$. DGGE profiles were analysed using the BioNumerics software, version 2.50 (Applied Maths, St-Martens-Latem, Belgium) and the resulting matrices were analysed by principal components analysis (CANOCO for Windows 4.5; Canoco, Wageningen, The Netherlands) ${ }^{(26)}$.
Total bacteria, Lactobacillus, Bacteroides, Clostridium cluster XIVa and Clostridium cluster IV were quantified as copy number/g by real-time PCR using specific primers and PCR conditions targeting $16 \mathrm{~S} \mathrm{rRNA}^{(27,28)}$. SYBR green PCR amplifications were performed in an iCycler iQ5 Real-Time Detection System (BioRad) associated with the iCycler Optical System Interface software (version 2; BioRad). The reaction mixture contained $2 \times \mathrm{SYBR}$ green PCR Mix (BioRad), $0.5 \mu \mathrm{M}$ of each primer, and either $5 \mu \mathrm{l}$ of ten times-diluted template DNA or water. For quantification of Bifidobacterium the recA gene was used as the target for real-time $\mathrm{PCR}^{(29)}$.

\section{Determination of myeloperoxidase activity}

Myeloperoxidase activity was measured according to the method of Stucchi et al. ${ }^{(30)}$.

\section{Oxidation damage}

DNA oxidative damage in the colon mucosa was assessed using two methods: the comet assay, on samples treated with a bacterial repair enzyme (formamidopyrimidine glycosylase; gift of A. R. Collins, University of Oslo, Norway $)^{(31)}$, and the HPLC determination of 8-hydroxy-2'deoxyguanosine levels ${ }^{(32)}$.

\section{Proliferative activity}

Colon mucosa proliferation was evaluated by counting nuclei immunoreactive to the proliferating cell nuclear antigen in at least ten longitudinal crypt sections at $400 \times{ }^{(33)}$. Proliferative activity was expressed as the number of labelled cells/the number of cells in the crypt $\times 100$.

\section{Microarray analysis}

Total RNA was extracted using the RNeasy Midi kit (Qiagen, Milan, Italy). Equal amounts of RNA extracted from the colon mucosa of control rats ( $n$ 6) were pooled and used as a common reference. RNA from six rats for each experimental group (labelled with Cy5) were compared with the reference RNA (labelled with Cy3) (CyDye Mono-Reactive Dye Pack; Amersham, Cologno Monzese, Milan, Italy), using the indirect labelling method described by DeRisi (http://derisilab.ucsf. edu). The twelve hybridisations were performed on the Agilent Whole Rat Genome $4 \times 44 \mathrm{~K}$ microarrays (Agilent Technologies, Palo Alto, CA, USA), according to the Agilent protocol; images were scanned using the Agilent DNA microarray scanner (G2505B; Agilent Technologies). Image analysis and initial quality control were performed using the Agilent Feature Extraction Software v9.5 and TIBCO SpotFire Decisionsite 9.0 (TIBCO Spotfire, Somerville, MA, USA).

For microarray data analysis, the text files were imported into R 2.5.1 using the Bioconductor 'limma' package 2.8.1 for statistical analysis ${ }^{(34,35)}$. Values for control spots and spots that did not meet the quality criteria were flagged. Quality criteria for inclusion included having a spot of at least thirty-five pixels, a median:mean ratio of at least 0.9 and a non-saturated spot intensity for both channels. Quality criteria for inclusion included the first two criteria for both channels, 
a non-saturated spot intensity and a signal well above background for at least one channel (background-corrected spot intensity larger than 2.6 times the SD of the measured background). The background-corrected intensities of all microarrays were normalised using the locally weighted scatterplot smoothing (LOESS) algorithm ${ }^{(36,37)}$. A linear model was then fitted to the intensities for each reporter, and treated rats were compared with controls using an unpaired moderated $t$ test using the 'limma' functions lmfit and eBayes ${ }^{(38)}$. Benjamini-Hochberg-adjusted $P$ values were used to correct for multiple testing. All Agilent reporters were re-annotated to ensure reporter specificity $^{(39)}$ and to optimise recognition by the pathway procedures. All non-specific reporters were included in the analysis step but were flagged to allow for correct interpretation.

Biological pathway analysis was performed using the GenMAPP/MAPPFinder software tandem ${ }^{(40)}$ (http://www. genmapp.org; University of California at San Francisco, San Francisco, CA, USA). For GenMAPP, the 'Rn_Std_ 20070817 ' gene database and the 'Rn_20080619' pathway set were used. MAPPFinder analyses for pathways enrichment were performed for both diets and for up- and down-regulated genes separately. MAPPFinder selection criteria were set at $P<0.05$ (Benjamini-Hochberg-adjusted) and $>1 \cdot 1$-fold change in the relevant direction. Only pathways showing a permute $P<0.05$ and a positive (enrichment) $z$-score were selected.

\section{$R T-P C R$}

For first-strand cDNA synthesis, $1 \mu \mathrm{g}$ of total RNA from each sample was reverse-transcribed. Primers were designed on the basis of the rat GenBank sequences for cyclo-oxygenase-2 (COX2), inducible NO synthase, IL1 $\beta$, TNF $\alpha$ and interferon $\gamma$. $\beta$-Actin was co-amplified as the reference ${ }^{(41)}$. Primer sequences are available on request.

\section{Statistical analyses}

Statistical analyses were conducted using GraphPad Prism software (GraphPad Software, Inc., La Jolla, CA, USA). Differences on faecal water content, myeloperoxidase activity, oxidative DNA damage, cell proliferation and RT-PCR measurements were analysed by one-way ANOVA followed by post hoc Tukey's multiple-range test, whereas the non-parametric Mann-Whitney test was used to analyse real-time PCR for Bacteroides fragilis spp. data. Results are presented as mean values with their standard errors. Significance was assigned at $P<0 \cdot 05$.

\section{Results}

During the feeding period, food intake and body-weight gain were similar among the three experimental groups; at the time of killing, no animals presented macroscopic intestinal bleeding. However, histological examination showed signs of inflammation and mild mucosal injury in all rats. The colitis score was significantly lower in rats treated with Marie Ménard apples compared with controls $(P<0 \cdot 05)$. Values were as follows: 1.2 (SEM 0.8$)$ in the control diet group, 1.0 (SEM 0.6) in rats fed lyophilised powder from Golden Delicious apples and 0.43 (SEM 0.4) in rats fed 7.6\% lyophilised powder from Marie Ménard apples.

Compared with controls, the severity of diarrhoea, evaluated as percentage of faecal water, was significantly reduced in rats fed lyophilised Marie Ménard apples, whereas rats fed lyophilised Golden Delicious apples had some improvements that did not reach statistical significance (Table 3).

To determine the dietary impact of the different diets on intestinal microbiota, total faecal bacteria and predominant intestinal bacterial populations were analysed at the end of the treatments by $16 \mathrm{~S}$ rRNA gene-based real-time PCR and DGGE. Real-time PCR indicated variations in the number of bacteria and population sizes of Bifidobacterium, Lactobacillus, Clostridium cluster XIVa and Clostridium cluster IV which were not significantly different among groups (data not shown). However, animals fed lyophilised Marie Ménard apples had significantly lower numbers of bacteria belonging to the $B$. fragilis group compared with those fed lyophilised Golden Delicious apples $(P=0.011)$ and controls $(P=0.051)$ (Fig. 1(a)). The real-time PCR data were confirmed by principal components analysis of the DGGE profiles (Fig. 1(b)), showing that Marie Ménard

Table 3. Effect of treatments with different lyophilised apples on diarrhoea (expressed as \% faecal water content), myeloperoxidase activity, oxidative DNA damage (expressed as \% of damaged DNA migrating in the cell tail after digestion of DNA with formamidopyrimidine glycosylase (FPG) or as 8-oxodG: $\mathrm{dG} \times 10^{-6}$ ratio) and cell proliferation (expressed as labelling index)

(Mean values with their standard errors)

\begin{tabular}{|c|c|c|c|c|c|c|}
\hline & \multicolumn{2}{|c|}{ Controls } & \multicolumn{2}{|c|}{$\begin{array}{l}\text { Rats fed Golden } \\
\text { Delicious apples }\end{array}$} & \multicolumn{2}{|c|}{$\begin{array}{l}\text { Rats fed Marie } \\
\text { Ménard apples }\end{array}$} \\
\hline & Mean & SEM & Mean & SEM & Mean & SEM \\
\hline Faecal water content (\%) & $87 \cdot 3$ & $2 \cdot 3$ & $77 \cdot 2$ & $4 \cdot 0$ & $80 \cdot 9^{*}$ & 1.5 \\
\hline Myeloperoxidase activity (U/g tissue) & $3 \cdot 6$ & 0.3 & $3 \cdot 0$ & 0.2 & $2 \cdot 2^{*} \dagger$ & 0.2 \\
\hline \multicolumn{7}{|l|}{ FPG-sensitive sites } \\
\hline$\%$ DNA in comet tail & $22 \cdot 8$ & $2 \cdot 2$ & $18 \cdot 8$ & $1 \cdot 4$ & $19 \cdot 7$ & $2 \cdot 0$ \\
\hline 8-oxodG:dG $\times 10^{-6}$ & $8 \cdot 4$ & $1 \cdot 0$ & $9 \cdot 7$ & $1 \cdot 2$ & $10 \cdot 1$ & 1.9 \\
\hline Labelling index & $45 \cdot 9$ & $2 \cdot 8$ & $33 \cdot 9^{*}$ & 1.9 & $39 \cdot 3$ & $2 \cdot 3$ \\
\hline
\end{tabular}

${ }^{*}$ Mean value was significantly different from that of the control group $(P<0.05)$.

$\dagger$ Mean value was significantly different from that of the rats fed lyophilised Golden Delicious apples. 

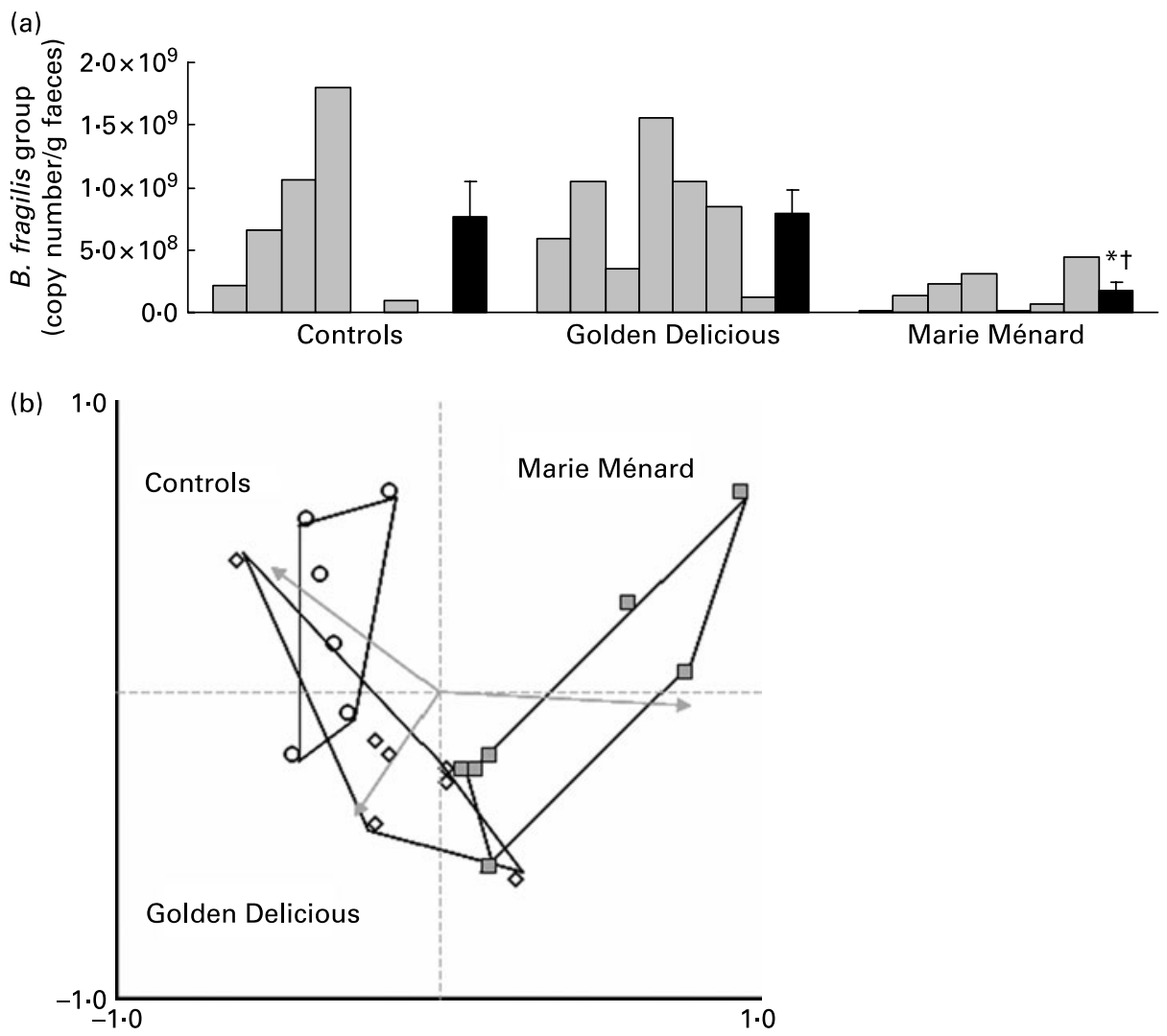

Fig. 1. (a) Real-time PCR for Bacteroides fragilis spp. from faeces of rats fed different diets, at the end of the treatment period. ( $\square$ ), Individual values; $(\boldsymbol{\square})$, mean values, with standard errors represented by vertical bars. ${ }^{*}$ Mean value was significantly different from that of the Golden Delicious apple-fed group $(P=0.011$; Mann-Whitney test). $†$ Mean value was marginally different from that of the control group $(P=0.051$; Mann-Whitney test). (b) Principal components analysis of the $B$. fragilis-group-specific denaturing gradient gel electrophoresis profiles in the three different dietary groups: controls $(O)$; Marie Ménard apple-fed rats $(\square)$; Golden Delicious apple-fed rats $(\diamond)$. Each symbol represents an individual faecal sample. $\rightarrow$, First three principal components. The Eigen value of the $x$ axis is 0.43 ; the Eigen value of the $y$ axis is 0.231 .

apple-fed rats had a distinct faecal Bacteroides population compared with the other two groups.

The myeloperoxidase activity in the colon mucosa, a marker of inflammatory cell infiltration, was significantly reduced in rats fed lyophilised Marie Ménard apples, compared with control rats and rats fed Golden Delicious apples (Table 3). Feeding lyophilised Golden Delicious apples reduced colon proliferative activity significantly $(P<0 \cdot 05)$, and Marie Ménard apples had a borderline effect $(P=0 \cdot 1)$, compared with the control diet (Table 3).

Oxidative DNA damage in the colon mucosa was not altered by the administration of either apple variety, measured with single cell electrophoresis (comet assay) or as the 8-hydroxy-2'-deoxyguanosine:2-deoxyguanosine ratio in DNA (Table 3).

Gene expression profiling revealed a large number of differentially expressed genes in rats fed lyophilised apples, relative to the control group (8492 and 11230 in rats fed Golden Delicious and Marie Ménard apples, respectively). Most differentially expressed genes (6945) were shared between the two apple treatments, whereas 1547 were exclusively modulated by Golden Delicious and a larger number (4285) by Marie Ménard apples. In the animals fed lyophilised apples, analysis of gene expression data with GenMapp/MAPP Finder programs identified several differentially expressed pathways (Table 4) relative to control: specifically, PG pathways and mitogen-activated protein kinase (MAPK) signalling were down-regulated. The TNF $\alpha-N F-\kappa B$ pathway was downregulated only in rats fed lyophilised Marie Ménard apples. The list of enriched pathways and the graphical representations showing the effect of both diets on gene expression in each pathway are available online at http://www.bigcat. unimaas.nl/public/data/GenMAPP/Apples

RT-PCR showed a significant reduction in $\operatorname{COX} 2(P<0.05)$ and inducible NO synthase- 2 gene expression $(P<0.01)$ in the colon mucosa of rats fed lyophilised Marie Ménard apples compared with controls (Fig. 2). The interferon $\gamma$ gene expression tended to be lower in rats fed Marie Ménard apples $(P=0.057)$ (Fig. 2), whereas TNF $\alpha$ and IL1 $\beta$ expression was similar in the three experimental groups.

\section{Discussion}

Several studies have suggested that phytochemicals contained in apples can prevent some important human diseases. In vitro ${ }^{(42)}$ and in vivo experiments ${ }^{(43)}$ suggest that apple extracts may have a protective role against colorectal cancer. Also, apple polyphenols can alleviate the symptoms of allergic rhinitis $^{(44)}$ and reduce the risk of type 2 diabetes ${ }^{(45)}$.

Apples may contain high amounts of polyphenols, depending not only on the cultivar but also on the harvesting, storage and processing steps. The bioavailability of polyphenols 
Table 4. Results of the functional analysis performed using GenMAPP/MAPPFinder software*

\begin{tabular}{|c|c|c|c|c|}
\hline MAPP name & Number changed & Number measured & Number on MAPP & $z$-Score \\
\hline \multicolumn{5}{|l|}{ Golden Delicious apple-treated rats } \\
\hline \multicolumn{5}{|l|}{ Pathways up-regulated } \\
\hline Rn_GPCRDB_Other & 19 & 43 & 62 & $4 \cdot 395$ \\
\hline Rn_Calcium_regulation_in_cardiac_cells & 38 & 130 & 150 & 3.269 \\
\hline Rn_Hedgehog_Netpath_10 & 8 & 17 & 20 & 3.054 \\
\hline Rn_Biogenic_Amine_Synthesis & 5 & 12 & 15 & $2 \cdot 08$ \\
\hline Rn_GPCRDB_Class_A_Rhodopsin-like & 27 & 105 & 144 & 1.969 \\
\hline \multicolumn{5}{|l|}{ Pathways down-regulated } \\
\hline Rn_Alpha6-Beta4-Integrin_NetPath_1 & 23 & 55 & 64 & 3.999 \\
\hline Rn_MAPK_signaling_pathway_KEGG & 38 & 120 & 136 & $3 \cdot 159$ \\
\hline Rn_HSP70_and_Apoptosis & 9 & 18 & 18 & $3 \cdot 134$ \\
\hline Rn_Cholesterol_Biosynthesis & 7 & 14 & 15 & $2 \cdot 761$ \\
\hline Rn_G13_Signaling_Pathway & 9 & 20 & 24 & $2 \cdot 747$ \\
\hline Rn_Fatty_Acid_Beta_Oxidation_Meta_BiGCaT & 11 & 27 & 31 & $2 \cdot 644$ \\
\hline Rn_Steroid_Biosynthesis & 4 & 7 & 11 & $2 \cdot 419$ \\
\hline Rn_Prostaglandin_synthesis_regulation & 11 & 29 & 32 & 2.363 \\
\hline Rn_Apoptosis & 22 & 71 & 82 & $2 \cdot 257$ \\
\hline Rn_Glycogen_Metabolism & 5 & 11 & 15 & 2.071 \\
\hline Rn_Fas_Pathway_and_Stress_Induction_ & 12 & 35 & 38 & $2 \cdot 06$ \\
\hline \multicolumn{5}{|l|}{ Marie Ménard apple-treated rats } \\
\hline \multicolumn{5}{|l|}{ Pathways up-regulated } \\
\hline Rn_GPCRDB_Other & 24 & 43 & 62 & $4 \cdot 762$ \\
\hline Rn_Ribosomal_Proteins & 24 & 63 & 81 & 2.486 \\
\hline Rn_Synthesis_and_Degradation_of_Ketone_Bodies & 3 & 4 & 4 & $2 \cdot 329$ \\
\hline Rn_Calcium_regulation_in_cardiac_cells & 43 & 130 & 150 & $2 \cdot 262$ \\
\hline Rn_Monoamine_GPCRs & 11 & 26 & 34 & $2 \cdot 084$ \\
\hline Rn_GPCRDB_Class_B_Secretin-like & 6 & 12 & 13 & 2.03 \\
\hline Rn_Biogenic_Amine_Synthesis & 6 & 12 & 15 & 2.03 \\
\hline \multicolumn{5}{|l|}{ Pathways down-regulated } \\
\hline Rn_Prostaglandin_synthesis_regulation & 16 & 29 & 32 & $4 \cdot 149$ \\
\hline Rn_MAPK_signaling_pathway_KEGG & 42 & 120 & 136 & 3.22 \\
\hline Rn_Alpha6-Beta4-Integrin_NetPath_1 & 22 & 55 & 64 & 3.04 \\
\hline Rn_G13_Signaling_Pathway & 10 & 20 & 24 & $2 \cdot 886$ \\
\hline Rn_Apoptosis & 26 & 71 & 82 & $2 \cdot 778$ \\
\hline Rn_RNA_transcription_Reactome & 12 & 28 & 41 & 2.517 \\
\hline Rn_Androgen-Receptor_NetPath_2 & 31 & 92 & 101 & $2 \cdot 497$ \\
\hline Rn_Glycogen_Metabolism & 6 & 11 & 15 & 2.495 \\
\hline Rn_Insulin_Signaling & 39 & 124 & 155 & $2 \cdot 31$ \\
\hline Rn_T-Cell-Receptor_NetPath_11 & 33 & 103 & 122 & $2 \cdot 24$ \\
\hline Rn_IL-6_NetPath_18 & 28 & 85 & 97 & $2 \cdot 228$ \\
\hline Rn_Signaling_of_Hepatocyte_Growth_Factor_Receptor & 11 & 27 & 33 & $2 \cdot 208$ \\
\hline Rn_EGFR1_NetPath_4 & 44 & 145 & 166 & $2 \cdot 183$ \\
\hline Rn_HSP70_and_Apoptosis & 8 & 18 & 18 & $2 \cdot 174$ \\
\hline Rn_B_Cell_Receptor_NetPath_12 & 40 & 132 & 146 & 2.065 \\
\hline Rn_TNF-alpha-NF-kB_NetPath_9 & 41 & 136 & 159 & $2 \cdot 053$ \\
\hline Rn_Fas_Pathway_and_Stress_Induction & 13 & 35 & 38 & 2.009 \\
\hline
\end{tabular}

GPCRDB, G Protein-Coupled Protein Receptor Database; MAPK, mitogen-activated protein kinase; KEGG, Kyoto Encyclopedia of Genes and Genomes; HSP, heat shock protein; BiGCaT, Bioinformatics Group; GPCRs, G protein-coupled protein receptors; EGFR1, epidermal growth factor receptor-1.

* Pathways significantly enriched ( $z$-score $>1.97$ ) are listed. The different columns show the number of genes differentially expressed compared with controls, the number of genes analysed and the number of genes listed in each map (http://www.genmapp.org; University of California at San Francisco, San Francisco, CA, USA).

differs greatly from one compound to another. A recent review of the ninety-seven published studies focused on the bioavailability of polyphenols ${ }^{(46)}$ reported that the plasma concentrations of total metabolites may range from 0 to $4 \mu \mathrm{mol} / 1$ after ingestion of $50 \mathrm{mg}$ of aglycone equivalents.

Regarding the high-molecular-weight compounds, although it has been reported that oligomers from apples are absorbed from the digestive tract and present in rat plasma ${ }^{(47)}$, polymeric proanthocyanidins are poorly absorbed in the gastrointestinal tract. However, it is possible that their beneficial effects at the intestinal level may not require efficient absorption through the gut be mediated by direct activity on the intestinal mucosa and/or indirect effect by varying the intestinal microbiota.

We tested the ability of lyophilised samples of two apple cultivars added to the diet to control colitis in an experimental inflammation model (HLA-B27 rats). The rationale for testing Marie Ménard apples, which are now used only for cider production, was based on their particularly high content of proanthocyanidins and hydroxycinnamic acids. It has been reported that proanthocyanidins modulate the expression of inflammatory genes such as COX2 and inducible NO synthase in murine macrophages stimulated with endotoxins ${ }^{(48,49)}$; moreover, rats fed Arabidopsis thaliana seeds containing proanthocyanidins show a down-regulation of genes associated with the inflammatory response in the colon mucosa ${ }^{(40)}$ Hydroxycinnamic acids, such as $p$-coumaric and ferulic acid, can also control the expression of COX2 in experimental inflammation in rodents ${ }^{(50,51)}$. Recently, we observed that lyophilised Marie Ménard apples administered to Wistar rats for 4 weeks down-regulated genes associated with 

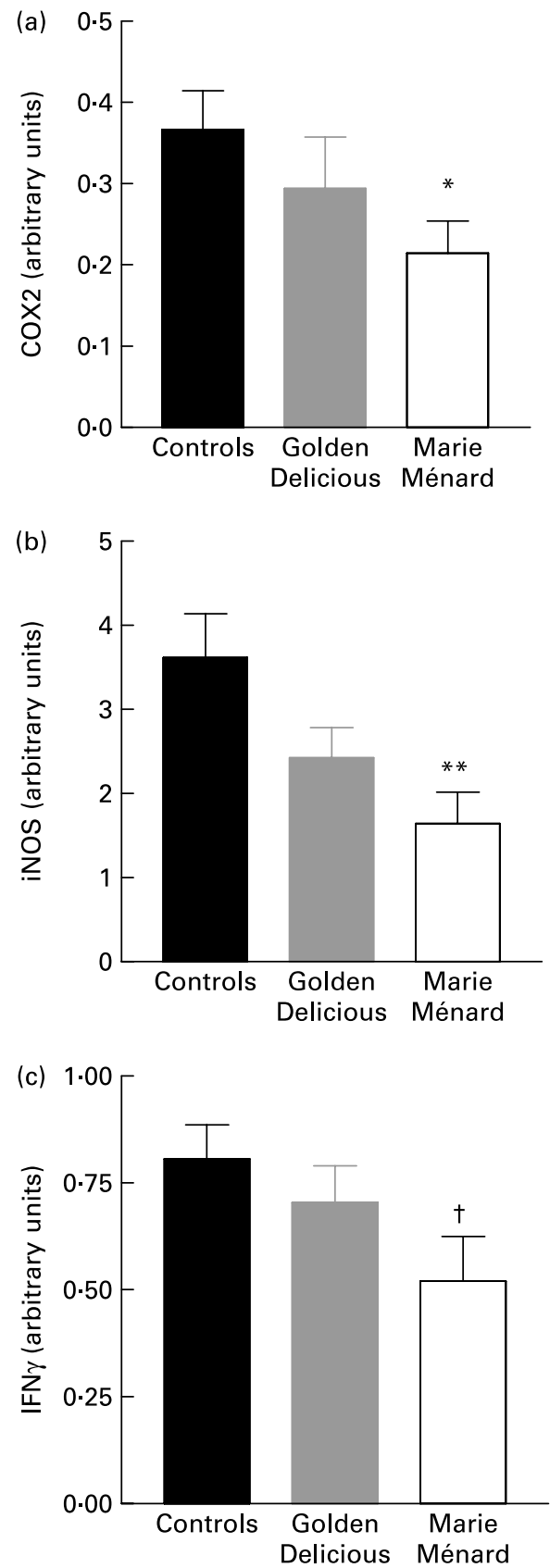

Fig. 2. Effect of lyophilised apple dietary treatments on the expression levels of selected pro-inflammatory genes: (a) cyclo-oxygenase-2 (COX2); (b) inducible NO synthase (iNOS); (c) interferon $\gamma$ (IFN $\gamma$ ). Values are means for six control rats, seven Golden Delicious apple-fed rats or seven Marie Ménard apple-fed rats, with standard errors represented by vertical bars. Mean value was significantly different from that of the control group: ${ }^{*} P<0.05,{ }^{* *} P<0.01$. † Mean value was marginally different from that of the control group $(P=0.057)$.

inflammatory responses in the colon mucosa ( $\mathrm{C}$ Luceri, unpublished results), suggesting a possible anti-inflammatory activity of this apple variety.

In the present study, rats fed lyophilised Marie Ménard apples had significantly fewer histological signs of colitis, less diarrhoea and a reduced colon mucosa myeloperoxidase activity. These effects were not related to the antioxidant activity of apple components, since DNA oxidative damage did not vary relative to controls by the administration of apples.

By gene expression profiling of the colon mucosa, we identified many down-regulated pathways, most of which were interconnected, such as the PG synthesis cascade, TNF $\alpha-$ NF- $\kappa \mathrm{B}$ signalling and the epidermal growth factor receptor (EGFR) pathway. COX2 is a key regulator of inflammation, pivotal to the induction of chemically induced colon cancer $^{(52)}$. Although the role of prostaglandins in intestinal inflammation is not totally clear, several eicosanoids increase in IBD and a correlation exists between their tissue levels and disease activity ${ }^{(53)}$; moreover 5-aminosalicylic acid is widely used in the treatment of IBD and is believed to act through the inhibition of the activation of transcription factor NF$\mathrm{\kappa B}^{(54)}$. TNF $\alpha$ is a pleiotropic cytokine that regulates many physiological processes, such as inflammation, proliferation and cell death, and exerts these effects by activating multiple downstream effectors including NF-кB. EGFR signalling has also emerged as an important therapeutic target. EGFR can be activated by several ligands and these interactions result in the activation of several downstream pathways including phosphoinositol-3-kinase-Akt; activated Akt phosphorylates a number of downstream targets and transcription factors such as $N F-\kappa B^{(55)}$. NF- $\kappa \mathrm{B}$ activates the transcription of a wide spectrum of pro-inflammatory genes with consequent production of cytokines, adhesion molecules and enzymes and is involved also in cell proliferation and apoptosis. Inhibitors of NF- $\mathrm{KB}$ activation have been shown to be potent anti-inflammatory agents ${ }^{(56,57)}$. Apple extracts, at high concentrations $(200-2000 \mathrm{nM})$, inhibit the $\mathrm{TNF} \alpha$ signal via $\mathrm{NF}-\kappa \mathrm{B}$ in human umbilical vein endothelial cells ${ }^{(58)}$ and in human breast cancer MCF-7 cells at a dose of $5 \mathrm{mg} / \mathrm{ml}^{(59)}$. Therefore, it is possible that some of the effects observed in the present study may be related to the inhibition of NF- $\mathrm{B}$ activation.

Improvement in colitis in HLA-B27 rats was associated with changes in the faecal microbiota; principally a reduction in B. fragilis group in animals fed Marie Ménard apples. This observation is consistent with previous findings showing that the addition of $B$. vulgatus to a cocktail of five anaerobic bacteria in germ-free HLA-B27 rats generates serious colitis, whereas administration of the same bacterial cocktail without B. vulgatus has no pro-inflammatory effect ${ }^{(60)}$. Another study in mice showed that $B$. fragilis exerts immunomodulatory activity on the intestine, inducing the transition from $\mathrm{CD}^{+}$ $\mathrm{CD} 45 \mathrm{Rb}^{\text {high }}$ to $\mathrm{CD}^{+} \mathrm{CD} 45 \mathrm{Rb}^{\text {low }}$ subsets through polysaccharide A activity ${ }^{(61)}$. In our experiments, the anti-inflammatory activity of Marie Ménard apples was associated with reduced $B$. fragilis group in the faeces. The interaction of Bacteriodes with intestinal inflammatory processes is complex and probably depends on the particular inflammation model studied. However, faecal Bacteriodes variations could influence intestinal inflammation.

The aim of the present study was to test a possible antiinflammatory effect of the whole apple added to the diet as lyophilised powder. The experimental approach mimics the effect of adding whole apple fruit to the human diet. This approach did not allow identification of single bioactive components. The effects we observed are probably due to a combination of different phytochemicals, including polyphenols, stilbenoids, lignans, triterpenoids and other constituents such as starches or fibres. We do not know at 
present which specific components of the different apple varieties are responsible for the anti-inflammatory effects. However, we did observe a strong effect in animals fed Marie Ménard apples. Considering that the main differences between the two apple cultivars are related to the proanthocyanidin and hydroxycinnamic acid content, we can infer that these compounds are probably the mediators of the anti-inflammatory effect.

In conclusion, the administration of Marie Ménard apples, now used only in the manufacturing of cider, ameliorates spontaneous colon inflammation in HLA-B27 transgenic rats. If effective also in humans, a simple treatment with lyophilised extracts of specific apple varieties might improve the quality of life of millions of individuals affected by IBD.

\section{Acknowledgements}

The authors thank Val-de-Vire Bioactives for their kind gift of apple fibres, G. Le Bail for lyophilised apple preparation, Professor Alessandra Renieri and Dr Filomena Papa for technical support for the scanning of microarray images, and Mary C. Forrest for linguistic revision of the manuscript.

The present study was supported by the European Network of Excellence in Nutrigenomics, NuGO (FOOD-CT-2004506360) (P. D., C. C., E. B. and C. L.), the European Union programme FLAVO (FOOD-CT-2004-513960) (V. P.), the Ministero dell'Università e della Ricerca Scientifica e Tecnologica, Italy, and the University of Florence, Italy. S. G. was partly funded by the Nutrigenomics Consortium of the Top Institute Food and Nutrition and by the Dutch BSIK Fund.

C. C., C. L. and P. D. conceived of and designed the experiments. The following authors performed the experiments: C. L. and E. B. (diets and animal treatment); C. L. (microarray and RT-PCR); E. B. and M. L. (8-hydroxy-2'-deoxyguanosine); L. G. and V. P. (comet assay); L. M. (histopathology); C. C., R. M. and E. G. Z. (faecal microbiota analysis); G. C., A. P. F. and M. S. (proliferative activity); C. M. G. C. R. and A. B. (apple powder production and characterisation). The statistics were analysed by S. T.; C. C., S. G., L. E. and C. T. E. analysed the bioinformatics. C. C., C. L. and P. D. wrote the paper.

C. C. and C. L. contributed equally to the present study.

The authors state that there are no conflicts of interest.

\section{References}

1. Loftus EV Jr (2004) Clinical epidemiology of inflammatory bowel disease: incidence, prevalence, and environmental influences. Gastroenterology 126, 1504-1517.

2. Xavier RJ \& Podolsky DK (2007) Unravelling the pathogenesis of inflammatory bowel disease. Nature 448, 427-434.

3. Jowett SL, Seal CJ, Pearce MS, et al. (2004) Influence of dietary factors on the clinical course of ulcerative colitis: a prospective cohort study. Gut 53, 1479-1484.

4. Yamamoto T, Nakahigashi M, Umegae S, et al. (2005) Impact of elemental diet on mucosal inflammation in patients with active Crohn's disease: cytokine production and endoscopic and histological findings. Inflamm Bowel Dis 11, 580-588.

5. Shah S (2007) Dietary factors in the modulation of inflammatory bowel disease activity. Med Gen Med 9, 60 .

6. Biesalski HK (2007) Polyphenols and inflammation: basic interactions. Curr Opin Clin Nutr Metab Care 10, 724-728.
7. Smith AH \& Mackie RI (2004) Effect of condensed tannins on bacterial diversity and metabolic activity in the rat gastrointestinal tract. Appl Envirion Microbiol 70, 1104-1115.

8. Dolara P, Luceri C, De Filippo C, et al. (2005) Red wine polyphenols influence carcinogenesis, intestinal microflora, oxidative damage and gene expression profiles of colonic mucosa in F344 rats. Mutat Res 591, 237-246.

9. Gaudio E, Taddei G, Vetuschi A, et al. (1999) Dextran sulfate sodium (DSS) colitis in rats: clinical, structural, and ultrastructural aspects. Dig Dis Sci 44, 1458-1475.

10. Hammer RE, Maika SD, Richardson JA, et al. (1990) Spontaneous inflammatory disease in transgenic rats expressing HLA-B27 and human $\beta 2 \mathrm{~m}$ : an animal model of HLA-B27associated human disorders. Cell 63, 1099-1112.

11. Taurog JD, Richardson JA, Croft JT, et al. (1994) The germfree state prevents development of gut and joint inflammatory disease in HLA-B27 transgenic rats. J Exp Med 180, 2359-2364.

12. Rath HC, Wilson KH \& Sartor RB (1999) Differential induction of colitis and gastritis in HLA-B27 transgenic rats selectively colonized with Bacteroides vulgatus or Escherichia coli. Infect Immun 67, 2969-2974.

13. Hoentjen F, Tonkonogy SL, Qian BF, et al. (2007) CD4(+) $\mathrm{T}$ lymphocytes mediate colitis in HLA-B27 transgenic rats monoassociated with nonpathogenic Bacteroides vulgatus. Inflamm Bowel Dis 13, 317-324.

14. Guyot S, Marnet N, Sanoner P, et al. (2001) Direct thiolysis on crude apple materials for high-performance liquid chromatography characterization and quantification of polyphenols in cider apple tissues and juices. Methods Enzymol 335, 57-70.

15. Hach CC, Brayton SV \& Kopelove AB (1985) A powerful Kjeldahl nitrogen method using peroxymonosulfuric acid. J Agric Food Chem 33, 1117-1123.

16. Renard CMGC (2005) Variability in cell wall preparations: quantification and comparison of common methods. Carbohydr Polym 60, 515-522.

17. Batey JL (1982) Starch analysis using thermostable $\alpha$-amylases. Starch/Stärke 34, 125-128.

18. Ella Missang C, Renard CMGC, Baron A, et al. (2001) Changes in the pectin fraction of bush butter (Dacryodes edulis (G. Don) H.J. Lam) fruits during ripening. J Sci Food Agric 81, 781-789.

19. Caderni G, Luceri C, Lancioni L, et al. (1998) Slow-release pellets of sodium butyrate increase apoptosis in the colon of rats treated with azoxymethane, without affecting aberrant crypt foci and colonic proliferation. Nutr Cancer 30, 175-181.

20. Zoetendal EG, Heilig HG, Klaassens ES, et al. (2006) Isolation of DNA from bacterial samples of the human gastrointestinal tract. Nat Protocol 1, 870-873.

21. Heilig HG, Zoetendal EG, Vaughan EE, et al. (2002) Molecular diversity of Lactobacillus spp. and other lactic acid bacteria in the human intestine as determined by specific amplification of 16S ribosomal DNA. Appl Environ Microbiol 68, 114-123.

22. Satokari RM, Vaughan EE, Akkermans AD, et al. (2001) Polymerase chain reaction and denaturing gradient gel electrophoresis monitoring of fecal Bifidobacterium populations in a prebiotic and probiotic feeding trial. Syst Appl Microbiol 24, 227-231.

23. Pang X, Ding D, Wei G, et al. (2005) Molecular profiling of Bacteroides spp. in human feces by PCR-temperature gradient gel electrophoresis. J Microbiol Methods 61, 413-417.

24. Muyzer G, de Waal EC \& Uitterlinden AG (1993) Profiling of complex microbial populations by denaturing gradient gel electrophoresis analysis of polymerase chain reaction-amplified genes coding for 16S rRNA. Appl Environ Microbiol 59, $695-700$

25. Sanguinetti CJ, Neto D \& Simpson AJG (1994) Rapid silver staining and recovery of PCR products separated on polyacrylamide gels. Biotechniques 17, 915-919. 
26. Leps J \& Smilauer P (2003) Multivariate Analysis of Ecological Data using CANOCO, Kindle edition. Cambridge: Cambridge University Press.

27. Requena T, Burton J, Matsuki T, et al. (2002) Identification, detection, and enumeration of human Bifidobacterium species by PCR targeting the transaldolase gene. Appl Environ Microbiol 68, 2420-2427.

28. Matsuki T, Watanabe K, Fujimoto J, et al. (2004) Use of $16 \mathrm{~S}$ rRNA gene-targeted group-specific primers for real-time PCR analysis of predominant bacteria in human feces. Appl Environ Microbiol 70, 7220-7228.

29. Masco L, Vanhoutte T, Temmerman R, et al. (2007) Evaluation of real-time PCR targeting the 16S rRNA and recA genes for the enumeration of bifidobacteria in probiotic products. Int $J$ Food Microbiol 113, 351-357.

30. Stucchi AF, Shofer S, Leeman S, et al. (2000) NK-1 antagonist reduces colonic inflammation and oxidative stress in dextran sulfate-induced colitis in rats. Am J Physiol Gastrointest Liver Physiol 279, G1298-G1306.

31. Giovannelli L, Decorosi F, Dolara P, et al. (2003) Vulnerability to DNA damage in the aging rat substantia nigra: a study with the comet assay. Brain Res 969, 244-247.

32. Lodovici M, Casalini C, Cariaggi R, et al. (2000) Levels of 8-hydroxydeoxyguanosine as a marker of DNA damage in human leukocytes. Free Radical Biol Med 28, 13-17.

33. Femia AP, Luceri C, Dolara P, et al. (2002) Antitumorigenic activity of the prebiotic inulin enriched with oligofructose in combination with the probiotics Lactobacillus rhamnosus and Bifidobacterium lactis on azoxymethane-induced colon carcinogenesis in rats. Carcinogenesis 23, 1953-1960.

34. Smyth GK (2005) Linear models for microarray data. In Bioinformatics and Computational Biology Solutions Using $R$ and Bioconductor, pp. 397-420 [R Gentleman, VJ Carey and W Huber, et al., editorss]. New York: Springer-Verlag.

35. Smyth GK, Yang YH \& Speed TP (2003) Statistical issues in cDNA microarray data analysis. Methods Mol Biol 224, 111-136.

36. Bolstad BM, Irizarry RA, Astrand M, et al. (2003) A comparison of normalization methods for high density oligonucleotide array data based on variance and bias. Bioinformatics 19, 185-193.

37. Yang YH, Dudoit S, Luu P, et al. (2002) Normalization for cDNA microarray data: a robust composite method addressing single and multiple slide systematic variation. Nucleic Acids Res 30, e15.

38. Smyth GK (2004) Linear models and empirical Bayes methods for assessing differential expression in microarray experiments. Stat Appl Genet Mol Biol 3, article 3.

39. Gaj S, van Erk A, van Haaften RI, et al. (2007) Linking microarray reporters with protein functions. BMC Bioinformatics 8, 360 .

40. Luceri C, Giovannelli L, Pitozzi V, et al. (2008) Liver and colon DNA oxidative damage and gene expression profiles of rats fed Arabidopsis thaliana mutant seeds containing contrasted flavonoids. Food Chem Toxicol 46, 1213-1220.

41. Luceri C, Caderni G, Sanna A, et al. (2002) Red wine and black tea polyphenols modulate the expression of cycloxygenase-2, inducible nitric oxide synthase and glutathione-related enzymes in azoxymethane-induced F344 rat colon tumors. J Nutr 132, $1376-1379$.

42. Veeriah S, Hofmann T, Glei M, et al. (2007) Apple polyphenols and products formed in the gut differently inhibit survival of human cell lines derived from colon adenoma (LT97) and carcinoma (HT29). J Agric Food Chem 55, 2892-2900.

43. Gossé F, Guyot S, Roussi S, et al. (2005) Chemopreventive properties of apple procyanidins on human colon cancer-derived metastatic SW620 cells and in a rat model of colon carcinogenesis. Carcinogenesis 26, 1291-1295.

44. Enomoto T, Nagasako-Akazome Y, Kanda T, et al. (2006) Clinical effects of apple polyphenols on persistent allergic rhinitis: a randomized double-blind placebo-controlled parallel arm study. J Investig Allergol Clin Immunol 16, 283-289.

45. Song Y, Manson JE, Buring JE, et al. (2005) Associations of dietary flavonoids with risk of type 2 diabetes, and markers of insulin resistance and systemic inflammation in women: a prospective study and cross-sectional analysis. J Am Coll Nutr 24, 376-384.

46. Manach C, Williamson G, Morand C, et al. (2005) Bioavailability and bioefficacy of polyphenols in humans. I. Review of 97 bioavailability studies. Am J Clin Nutr 81, 230S-242S.

47. Shoji T, Masumoto S, Moriichi N, et al. (2006) Apple procyanidin oligomers absorption in rats after oral administration: analysis of procyanidins in plasma using the Porter method and high-performance liquid chromatography/tandem mass spectrometry. J Agric Food Chem 54, 884-892.

48. Hou DX, Masuzaki S, Hashimoto F, et al. (2007) Green tea proanthocyanidins inhibit cyclooxygenase-2 expression in LPSactivated mouse macrophages: molecular mechanisms and structure-activity relationship. Arch Biochem Biophys 460, 67-74.

49. Terra X, Valls J, Vitrac X, et al. (2007) Grape-seed procyanidins act as antiinflammatory agents in endotoxin-stimulated RAW 264.7 macrophages by inhibiting NFKB signaling pathway. J Agric Food Chem 55, 4357-4365.

50. Sudheer AR, Muthukumaran S, Devipriya N, et al. (2008) Influence of ferulic acid on nicotine-induced lipid peroxidation, DNA damage and inflammation in experimental rats as compared to N-acetylcysteine. Toxicology 243, 317-329.

51. Luceri C, Guglielmi F, Lodovici M, et al. (2004) Plant phenolic 4-coumaric acid protects against intestinal inflammation in rats. Scand J Gastroenterol 39, 1128-1133.

52. Müller-Decker K \& Fürstenberger G (2007) The cyclooxygenase-2-mediated prostaglandin signaling is causally related to epithelial carcinogenesis. Mol Carcinog 46, 705-710.

53. Hendel J \& Nielsen OH (1997) Expression of cyclooxygenase-2 mRNA in active inflammatory bowel disease. Am J Gastroenterol 92, 1170-1173.

54. Bantel H, Berg C, Vieth M, et al. (2000) Mesalazine inhibits activation of transcription factor NF- $\mathrm{BB}$ in inflamed mucosa of patients with ulcerative colitis. Am J Gastroenterol 95, 3452-3457.

55. Talapatra S \& Thompson CB (2001) Growth factor signaling in cell survival: implications for cancer treatment. J Pharmacol Exp Ther 298, 873-878.

56. Roman-Blas JA \& Jimenez SA (2008) Targeting NF-кB: a promising molecular therapy in inflammatory arthritis. Int Rev Immunol 27, 351-374.

57. Peterson JM \& Guttridge DC (2008) Skeletal muscle diseases, inflammation, and NF-кB signaling: insights and opportunities for therapeutic intervention. Int Rev Immunol 27, 375-387.

58. Yoon H \& Liu RH (2007) Effect of selected phytochemicals and apple extracts on NF- $\mathrm{KB}$ activation in human breast cancer MCF-7 cells. J Agric Food Chem 55, 3167-3173.

59. Davis PA, Polagruto JA, Valacchi G, et al. (2006) Effect of apple extracts on NF- $\kappa \mathrm{B}$ activation in human umbilical vein endothelial cells. Exp Biol Med 231, 594-598.

60. Rath HC, Wilson KH \& Sartor RB (1999) Differential induction of colitis and gastritis in HLA-B27 transgenic rats selectively colonized with Bacteroides vulgatus or Escherichia coli. Infect Immun 67, 2969-2974.

61. Mazmanian SK, Round JL \& Kasper DL (2008) A microbial symbiosis factor prevents intestinal inflammatory disease. Nature 453, 620-625. 